Accepted refereed manuscript of:

Coll-Planas L, Watchman K, Doménech S, McGillivray D, O'Donnell H \& Tolson D (2017) Developing evidence for football (soccer) reminiscence interventions with long-term care: a cooperative approach applied in Scotland and Spain, Journal of the American Medical Directors Association, 18 (4), pp. 355-360.

DOI: $\underline{10.1016 / j . j a m d a .2017 .01 .013}$

(C) 2017, Elsevier. Licensed under the Creative Commons AttributionNonCommercial-NoDerivatives 4.0 International

http://creativecommons.org/licenses/by-nc-nd/4.0/ 
Title:

\section{DEVELOPING EVIDENCE FOR FOOTBALL REMINISCENCE INTERVENTIONS WITHIN LONG-TERM CARE: A CO-OPERATIVE APPROACH APPLIED IN SCOTLAND AND SPAIN.}

Author names and affiliations: Laura Coll-Planas ${ }^{a, b}$, Karen Watchman ${ }^{c}$, Sara Doménech ${ }^{\mathrm{a}, \mathrm{b}}$, David McGillivray ${ }^{\mathrm{d}}$, Hugh O’Donnelle, Debbie Tolson ${ }^{\mathrm{c}}$.

a Fundació Salut i Envelliment (Foundation on Health and Ageing), Universitat Autònoma de Barcelona, Barcelona, Spain.

${ }^{b}$ Institute of Biomedical Research (IIB Sant Pau), Barcelona, Spain.

c School of Health, Nursing and Midwifery, University of the West of Scotland, UK.

d School of Media, Culture \& Society, University of the West of Scotland, Glasgow, UK.

e Department of Social Sciences, Media and Journalism, Glasgow Caledonian University, Glasgow, UK.

Karen Watchman and Sara Doménech contributed equally to the manuscript.

E-mail address of authors: Laura.Coll@uab.cat, karen.watchman@stir.ac.uk,

Sara.Domenech@uab.cat, David.McGillivray@uws.ac.uk,

H.ODonnell@gcu.ac.uk, Debbie.Tolson@uws.ac.uk

\section{Laura Coll-Planas}

Fundació Salut i Envelliment UAB

Casa Convalescència UAB

C/ Sant Antoni M. Claret 171, 4a planta 08041 Barcelona

(+34) 934335030

laura.coll@uab.cat

\section{Sara Doménech}

Fundació Salut i Envelliment UAB

Casa Convalescència UAB

C/ Sant Antoni M. Claret 171, 4a planta 08041 Barcelona (+34) 934335030

sara.domenech@uab.cat

Karen Watchman ${ }^{1}$ (Karen Watchman conducted this research at University of the West of Scotland)

Alzheimer Scotland Senior Lecturer in Dementia/Depute Director

Alzheimer Scotland Centre for Policy and Practice

University of West of Scotland 
School of Health, Nursing and Midwifery

1.21 Caird Building

Hamilton

ML3 0JB

Tel: +44 (0)1698 283100 Ext 8639

United Kingdom

${ }^{1}$ Karen Watchman (present address)

Senior Lecturer in Frailty, Ageing and Dementia

Senior Fellow Higher Education Academy

Pathfoot J13, Faculty of Health Sciences

University of Stirling, Stirling, FK9 4LA

Tel: +44 (0) 1786466387

Karen.Watchman@stir.ac.uk

David McGillivray

Chair in Event \& Digital Cultures

School of Media, Culture \& Society

University of the West of Scotland

High Street, Paisley, PA1 2BE

Tel 01418483220

United Kingdom

David.McGillivray@uws.ac.uk

\section{Hugh O'Donnell}

Professor of Language and Popular Culture

Department of Social Sciences, Media and Journalism,

Glasgow School for Business and Society

Glasgow Caledonian University,

Cowcaddens Road, Glasgow, G4 0BA

Tel: +441413313262

Glasgow, United Kingdom

H.ODonnell@gcu.ac.uk

Debbie Tolson

Alzheimer Scotland Professor of Dementia/Director

Alzheimer Scotland Centre for Policy and Practice

University of West of Scotland

School of Health, Nursing and Midwifery

1.21 Caird Building

Hamilton

ML3 0JB

Tel $+44(0) 1698283100$ Ext 8669

Debbie.tolson@uws.ac.uk

Corresponding author: Laura Coll-Planas, Laura.Coll@uab.cat

Fundació Salut i Envelliment UAB Casa Convalescència UAB C/ Sant Antoni M. Claret 171, 4a planta 08041 Barcelona. (+34) 934335030 
- Up to five keywords relevant to the content of the manuscript.

Dementia, long-term care, reminiscence, football, loneliness. 


\title{
Developing evidence for football (soccer) reminiscence interventions within long-term care: a co-operative approach applied in Scotland and Spain.
}

\author{
Abstract \\ Loneliness is a common experience within long-term care and, to promote wellbeing and \\ quality of life among people with dementia, it is important to draw upon a repertoire of \\ strategies that provide social stimulation, companionship and enjoyment. This paper describes \\ and reflects on a program of co-operative social participatory research which sought to \\ introduce football-focused (i.e. soccer-based) reminiscence based in four community settings \\ within Spain and Scotland. Findings are reported and inform an original conceptual model that \\ supports the introduction of sustainable approaches to the development of football-focused \\ reminiscence with and for people with dementia.
}




\section{Introduction}

Dementia is a major public health concern worldwide ${ }^{1,2}$. Dementia-related changes, which include impaired cognition, memory loss, communication difficulties and behavioral changes, can reduce an individual's confidence to participate and engage in social activities increasing the likelihood of isolation and loneliness. Loneliness is considered a geriatric syndrome and, surprisingly, its magnitude in long-term care facilities is not known but thought to be high ${ }^{3}$. However, feelings of uselessness and meaninglessness, which fuel loneliness, are frequently reported within long-term care settings ${ }^{4}$. Loneliness in people with dementia leads to faster cognitive decline, depression, poorer ability to make decisions, reduced physical activity and increased frailty $\frac{5.6}{}$. Interventions to address loneliness and social isolation within nursing homes have included volunteer visits to provide support, cognitive behavioral therapy, internet training, companion-type robots ${ }^{\underline{7} \cdot \underline{8}}$, animal-assisted therapy ${ }^{9}$, contact with children, pets and plants and humor therapy through clowns $\underline{10,11}$. While some of these specific innovations have been proven effective and may appeal to some residents, many older people long for human relationships and reciprocity in giving and receiving ${ }^{4,12}$, hence the importance of human interaction and psychosocial interventions within dementia care ${ }^{\frac{13}{3}}$. Systematic reviews, however, reveal an inconclusive evidence base in terms of the effectiveness of loneliness interventions, indicating that theoretically informed, group-based interventions which harness community resources yield the most promising results. This resonates with Coll-Planas et al's $\mathbf{1}^{\underline{14}}$ theory-driven work that promotes the use of social capital to alleviate loneliness among older people.

\section{Reminiscence}

Reminiscence is one of the most popular interventions in practice in nursing homes $\frac{15}{}$. Reminiscence therapy provides cognitive stimulation for people with dementia. It consists of 
those people thinking about their own past experiences to reactivate their personal past and maintains the subject's personal identity by presenting facilitating stimuli such as objects or pictures. Past memories linked to significant life moments act as therapeutic and guiding elements for people with dementia, providing a sense of belonging. Structured reminiscence around the experiences of a person's life involves the use of selected facilitating stimuli to evoke significant and personalized memories. Reminiscence therapy is also used to stimulate communicative interactions and minimize social isolation, encouraging interaction between participants $\underline{16}, \underline{17}, \underline{18}$.

Importantly, there is growing evidence indicating the therapeutic potential of reminiscence; a recent meta-analysis showed positive results for cognition and depressive symptoms $\frac{19}{}$. Moreover, some results also show favorable effects on dysphoria and agitation in residents with dementia, like those from the LEAP $\operatorname{program}^{20}$.

Individualized reminiscence in nursing home residents has been shown to be effective in several studies ${ }^{21}, \underline{22}$. One-to-one interventions allow a high commitment to person-centered care and life-story work. An alternative option is to provide group-based reminiscence interventions framed around a shared interest. In this regard, there has been a growing interest in sportsbased reminiscence topics within long-term care. Scottish football-focused (i.e. soccer-based) reminiscence projects have demonstrated enthusiasm from those with an interest in the sport to engage in community-based activities and groups, including large-scale reminiscence events at stadia $\underline{23}, \underline{24}$. In the USA, baseball-focused reminiscence is growing in popularity $\underline{25}$.

\section{Football: from a collective social memory to a reminiscence tool}

Historically, European football clubs and their associated cultural practices have played an important role, socially and culturally, in the lives of working-class men in particular. Football offers a means of enhancing social relationships, tying people together through reciprocal 
relations based on mutual identification and trust, alongside shared "memory" of certain signs, symbols, sounds and places. Football supporters have an emotional attachment to the place that their club plays at, providing an important social identity ${ }^{26}$, and an affectionate relationship to the ground that is regularly revisited ${ }^{27}$. The psychological importance of the football club to a town or city is a source of "topophilia - a love of place" $\underline{28}$. The tie is so strong because the ground and club provide a hugely significant and comforting social bond - where people can interact with like-minded individuals.

The appeal of football within both Spain and Scotland, the sociability of football spectatorship and its association with both private and collective memories make it an ideal vehicle through which to develop both sociable and potentially therapeutic reminiscence interventions.

\section{Project aim}

This paper describes a co-operative approach to developing a theoretical and practice-driven evidence base to inform the delivery of football-based reminiscence to older people with cognitive impairment in long-term care contexts.

The overall project aim was to develop a conceptual model for practice, drawing upon the football reminiscence implementation studies undertaken within Spain and Scotland, and enriched by theoretical frameworks.

\section{Methods}

The projects in Scotland and Spain were delivered between 2013 and 2015; both took a cooperative-inquiry approach and enabled sequential engagement in different study sites. Research teams based within Scotland and Spain worked co-operatively to share ideas, developed project methods, and compared experiences and findings related to introducing football reminiscence within four different settings. 


\section{Study sites}

In Spain, three study sites were engaged, each from a different city: one day hospital from Barcelona and two nursing homes from Valencia and Bilbao.

In Scotland, four geographically close urban care homes operated by the same service provider within the Lanarkshire area participated.

\section{Football reminiscence implementation guide}

Drawing on our previous research ${ }^{29}$ we developed a template for guidance in the delivery of community-based structured football reminiscence called "Principles and Practice Guide for Developing Football-Focused Reminiscence with People with Dementia”. (Appendix 1). This was used as a starting point for the project teams working in Spain and Scotland to adapt for local implementation and evaluation within the respective study sites.

For brevity we will report the three consecutive projects undertaken in Spain first, followed by the Scottish project. In practice there was an ongoing iterative and co-operative process of discussion between the Spanish and Scottish projects so that implementation lessons could be shared and adjustments made to improve practices for immediate benefit to recipients of the reminiscence sessions, rather than waiting until the end of the project.

\section{Implementation projects undertaken in Spain}

Three football-based reminiscence programs were conducted, one in each city, in Barcelona, Valencia and Bilbao.

Inclusion criteria: For each of the three Spanish sites, a maximum number of ten older adults per group was sought. Participants had to be aged 65 years and over, with mild cognitive impairment or mild to moderate dementia (Global Deterioration Scale 3, 4 or $5^{\frac{30}{}}$ ) and interested in football in order to be eligible for the Spanish study. Exclusion criteria were participants 
who did not understand Spanish, were not able to participate in a group dynamic (due to severe behavioral, sensory and/or mental disorders) and participants co-morbid with terminal illness.

Participants' characteristics: A total of 20 participants were recruited, five in Barcelona, eight in Valencia and seven in Bilbao. Three of the participants were women and 13 had a low educational level. Four participants were recruited in Valencia despite having no cognitive decline due to the difficulty of finding enough people fitting the profile with an interest in football and willing to participate in the chosen nursing home. Three former football players with cognitive impairment were included in Valencia $(n=2)$ and Bilbao $(n=1)$.

Characteristics of the program: A 12-week structured program comprising 11 weekly twohour football reminiscence sessions was delivered in the three settings. The program was tailored to the specificities of each of the three intervention sites. The final session at each site involved a visit to the local football stadium and/or football museum, specifically the stadium and museum from the local clubs: FC Barcelona, FC Valencia and Athletic Club de Bilbao.

Sessions were composed of reminiscence activities. After projecting images and audio related to the football, participants were encouraged to discuss their memories in a friendly atmosphere. A Life Story Book in relation to football was developed by the researchers with the support of the University of the West of Scotland. Part of each session was dedicated to working on the Life Story Book. Family members were asked to collaborate in developing the Life Story Book by providing pictures of different moments of the patient's life (childhood, adolescence and adulthood). In Bilbao, family members were invited to join the visits to the local football museum and stadium. The facilitator presented reminiscence memorabilia and a variety of football objects such as football cards, newspaper clippings, pennants and videos to trigger conversations. 
Football material was carefully chosen by the Spanish Federation of Associations of Former Football Players (FEAFV) in accordance with guidance provided by the University of the West of Scotland. The local FEAFV involved were Agrupació Barça Veterans (Barcelona), Asociación de Futbolistas Valencia CF (Valencia), and Asociación Exjugadores Athletic Club de Bilbao (Bilbao). Further stakeholders who collaborated in the design and delivery of the intervention included: healthy and active former football players from the local associations of former football players linked to the FEAFV who acted as volunteers providing their football experience and expertise during the sessions; former players with dementia from the local associations of former football players linked to the FEAFV who benefitted from the intervention while contributing with their personal football history to the group (in Valencia and Bilbao); a psychologist (from the centers in Bilbao and Valencia) and a researcher in Barcelona, all with experience of facilitating groups and trained in football-based reminiscence, who facilitated the sessions; other health professionals from the center, who observed the sessions and supported participants when needed.

Evaluation methods: Qualitative and quantitative data were collected to reflect the process and the impact of the program on those involved. As quantitative measures, validated scales related to cognition, behavior, function, communication and quality of life of participants with dementia, as well as caregiver's burden, were administered at the beginning and after finishing the intervention to assess their applicability, and to estimate the sample size of a future randomized controlled clinical trial.

The qualitative evaluation was based on observations and semi-structured interviews on opinions and experiences of the professionals, participants, volunteers and caregivers involved. Interviews were held after finishing the intervention and recorded for subsequent analysis. We applied the framework from Patton et al. $\frac{31}{}$ to evaluate the program according to: needs of the 
program, design, implementation, impact and continuity. Qualitative data were analyzed using content analysis.

\section{Implementation project undertaken in Scotland}

In Scotland, one program was conducted in one care home within the Lanarkshire area. For continuity, one facilitator was employed for the duration of the study.

Inclusion criteria: Inclusion criteria stipulated that the men were resident in one of four participating care homes owned by the same company, had a diagnosis of dementia, were able to consent to take part and expressed an interest and desire to attend.

Participants' characteristics: Eight male care-home residents with dementia were recruited, five of whom were regular attendees. They were aged between 72 and 89 . All had been resident in their respective care home for over six months.

Characteristics of the program: A 12-week structured reminiscence program was tested. This comprised weekly two-hour sessions delivered within one of the four participating nursing homes over a period of 11 weeks. The final week involved a group visit to Hampden Park, Scotland's national football stadium. Transport was provided for participants to travel from three other homes to the care home in which the program was delivered.

In addition to the trained facilitator, a care-home liaison physiotherapist and care-home liaison occupational therapist were in attendance each week providing a link between the research team and the care-home staff.

Retro football shirts and scarves were hung across the back of chairs and other artifacts were displayed as visual triggers in the reminiscence room. Structured activities were themed around Scottish, English and European football players, matches and associated factors such as match 
food and drink, and travel to games. Pies and Bovril, the staple fare of Scottish football matches, were provided and consumed at "half-time" each week.

Evaluation methods: Sessions were audio recorded and transcribed, interpretation was supported with use of documentary photographs and field notes were made by an independent observer. The facilitator and care-home staff kept a reflective log which family members were encouraged to write in to note any changes they observed in between the weekly sessions, including how often the football sessions were raised in conversation. As with the Spanish study, qualitative data were analyzed using content analysis. Quantitative data including care records consisting of falls data, sleep pattern, Malnutrition Universal Screening Tool (MUST), and medication records were scrutinized at the beginning and end of the 12 -week study period to determine evidence of impact on the residents' wellbeing and behavior.

\section{Building a model for practice}

Within the co-operative approach, a theoretically and practice-driven model for practice was built. The theoretical perspective was enriched by the Senses framework $\frac{32}{2}$ which recognizes the relational aspects within care and caring and the centrality of the person receiving care or community interventions ${ }^{33}$. This framework is focused on the creation of an environment in which older people, or, in our project, participants of the football reminiscence intervention, experience six senses, namely: sense of security, sense of belonging, sense of continuity, sense of purpose, sense of achievement and sense of significance.

The results gathered from the experiences in both countries contributed to building the model from a practical perspective.

\section{Ethical approval}

Ethical approval was sought for the respective study sites from the appropriate committees along with local management permissions. In Spain ethical approval was secured from the 
Comissió d'Ėtica en l'Experimentació Animal i Humana (CEEAH) de la Universitat Autònoma de Barcelona. In Scotland approval was secured from the University of the West of Scotland.

\section{Results}

\section{The Spanish project}

$\underline{\text { Table } 1}$ shows the results of the Spanish Project for participants. Professionals involved felt that they had acquired a new intervention tool that allowed them to gain a deeper knowledge of the participants, working with their strengths and helping to implement a more personcentered care. Family members reported an improvement in the relationships with their relatives with dementia. Former players who were enrolled as volunteers expressed satisfaction in being able to help with their knowledge and experience and were grateful to feel connected with other people and other realities. Former football players with dementia additionally felt needed and useful and able to contribute and help others.

\section{Legend of Table 1:}

Table 1. Results for participants of the Spanish and the Scottish projects.

\section{The Scottish project}

$\underline{\text { Table } 1}$ highlights how participants benefitted in multiple ways from intervention ${ }^{34}$.

\section{A model for practice}

The resulting conceptual model for practice is presented in Figure 1. It presents the collaborative partnership and the main mediating pathways identified as key factors to achieve successful results. Achievement of Nolan et al's ${ }^{32}$ Senses is a prerequisite to creating the conditions in which football reminiscence can be most helpful. For instance, this means that the individual feels safe and secure within the group, and has a sense of purpose (enjoyment) 
and achievement with affirming feedback from the facilitator recognized as important. Legend of Figure 1:

Figure 1. Model for practice: collaborative partnership and mediating factors.

\section{Discussion}

This paper describes and reflects on a program of co-operative social participatory research, which sought to introduce football-focused reminiscence into four long-term care settings within Scotland and Spain. Findings from each of the four projects are reported and compared and inform an original conceptual model that supports the introduction of sustainable approaches to the development of football-focused reminiscence with and for people with dementia.

In the implementation and delivery of the Spanish project, recruitment was difficult due to the specific target profile (mild to moderate dementia with an interest in football) and given the lower presence of men in long-term care. Nevertheless, the co-operation between former players and health professionals was key in complementing the knowledge and experience of football with that of dementia care. The atmosphere of camaraderie was determinant for the socialization process and for working with the strengths of participants with dementia. Finally, football was a useful tool to connect with enjoyment and feelings of belonging.

In the implementation and delivery of the Scottish Project, a key lesson was that the reminiscence facilitator required a combination of knowledge of football, dementia awareness, and interpersonal skills to involve men with different levels of cognitive ability within the group. This was required to promote the participation of all group members, particularly those less capable of responding verbally and quickly, while also conducting an enjoyable session, holding the men's attention and engaging them with impromptu banter. 
Regarding methods, the advantage of using co-operative inquiry $\frac{35}{5}$ and emergent actionorientated interventions was that the research teams could respond to the local context and learn implementation lessons that have given rise to an original conceptual model for practice. At the same time, working with different stakeholders was challenging when it came to agreeing and implementing a specific program, with different perspectives and purposes involved. However, this is a very good example of how a collaborative partnership has led to positive outcomes.

In terms of impacts, football-based reminiscence stimulated communicative interactions, cognitive abilities, improves mood and psychological wellbeing. Furthermore, it encouraged interaction between participants and provided a sense of belonging, thus minimizing social isolation and loneliness. In this vein, it supports but goes beyond person-centered care, since it is an example of a relationship-centered intervention as promoted through the Senses framework $\frac{32}{2}$. Therefore, it links into a sense of security (feeling safe in our existential being, safe places - stadia), a sense of belonging (football belonging - sense of home and familiarity), a purpose (enjoying football reminiscence), a sense of continuity ("I am still me"), achievement ("I can talk about football") and significance (feeling valued as a person).

The model for practice shows the collaborative partnership and mediating pathways and aims to complement the "Principles and Practice Guide for Developing Football-Focused Reminiscence with People with Dementia" (Appendix 1) in supporting practice and developing an evidence base for football reminiscence interventions within long-term care.

\section{Conclusion}

Loneliness is a common experience within long-term care and, to promote wellbeing and quality of life among people with dementia, it is important to draw upon a repertoire of strategies that provide social stimulation, companionship and enjoyment. Group-based football 
reminiscence interventions, as these projects have demonstrated, are feasible in a variety of long-term care settings including nursing homes, day care and community care and have the potential to bring people with dementia together to enjoy a shared and meaningful activity. The practice guide (Appendix 1) proved a useful starting point to shape local delivery approaches, and the new conceptual model offers a deeper consideration for long-term care professionals and applied researchers to further develop and deepen understanding of how such approaches might harness social capital within the community to alleviate loneliness in the most dependent and vulnerable members of their community.

Conflicts of interest: No conflicts of interest

\section{Financial support:}

The Scottish project was funded by Alzheimer Scotland Pilot Study Fund and conducted by a research team led from the University of the West of Scotland.

The Spanish project was promoted and financed by the Spanish Federation of Associations of Former Football Players (FEAFV) and led and coordinated by the Foundation for Health and Aging ( $\mathrm{FSiE}$ ) at the Universitat Autònoma de Barcelona (UAB) with the scientific advice of the University of the West of Scotland.

Acknowledgements: LCP conducted the Spanish project and published this paper within the PhD Program of Preventive Medicine and Public Health at the Universitat Autònoma de Barcelona. 


\section{References}

1. World Health Organization and Alzheimer's Disease International. Dementia. A Public Health Priority. Geneva: Alzheimer Disease International and World Health Organization. 2012 http://www.who.int/mental_health/publications/dementia_report_2012/en/

2. Pot AM, Petrea I. Improving dementia care worldwide: Ideas and advice on developing and implementing a National Dementia Plan. London, Bupa/ADI. 2013

3. Andrew N, Meeks S. Fulfilled preferences, perceived control, life satisfaction and loneliness in elderly long-term care residents. Aging and Mental Health. 2016;1-7.

4. Pitkala KH. Loneliness in Nursing Homes. J Am Med Dir Assoc. 2016;17:680-1.

5. Cacioppo JT, Hawkley LC. Perceived social isolation and cognition. Trends Cogn Sci. 2009;13:447-454.

6. Hawkley LC, Thisted RA, Cacioppo JT. Loneliness predicts reduced physical activity: cross-sectional \& longitudinal analysis. Health Psychol. 2009;28:354-363.

7. Bemelmans R, Gelderblom GJ, Jonker P, de Witte L. Socially assistive robots in elderly care: a systematic review into effects and effectiveness. J Am Med Dir Assoc. 2012;13:114-120.

8. Robinson H, Macdonald B, Kerse N, et al. The Psychosocial Effects of a Companion Robot: A Randomized Controlled Trial. J Am Med Dir Assoc. 2013;14:661-7.

9. Banks MR, Willoughby LM, Banks WA. Animal-assisted therapy and loneliness in nursing homes: use of robotic versus living dogs. J Am Med Dir Assoc. 2008;9:173-7.

10. Dickens AP, Richards SH, Greaves CJ, Campbell JL. Interventions targeting social isolation in older people: a systematic review. BMC Public Health. 2011;11:647.

11. Coll-Planas L, Nyqvist F, Puig T, et al. Social capital interventions targeting older people and their impact on health: a systematic review. J Epidemiol Community Health. 2016;1-10.

12. Vernooij-Dassen M, Leatherman S, Olde-Rikkert M. Quality of care in frail older people: the fragile balance between receiving and giving. BMJ. 2011;342-403.

13. Moniz-Cook E, Vernooij-dassen M, Woods R, et al. A European Consensus on outcome measures for psychosocial intervention research in dementia care. Aging \& Mental Health. 2008;12:14-29.

14. Coll-Planas L, Del Valle-Gómez G, Bonilla P, et al. Promoting social capital to alleviate loneliness and improve health among older people in Spain. Health Soc Care Community. 2017;25:145-157.

15. Cotelli M, Manenti R, Zanetti O, et al. Reminiscence therapy in dementia: A Review. Maturitas. 2012;72:203-5.

16. Bahar-Fuchs A, Clare L, Woods B. Cognitive training and cognitive rehabilitation for mild to moderate Alzheimer's disease and vascular dementia. Cochrane Database of Systematic Reviews. 2013;6:CD003260.

17. Woods B, Aguirre E, Spector A, Orrell M. Cognitive stimulation to improve cognitive functioning in people with dementia. Cochrane Database of Systematic Reviews. 2012;2:CD005562.

18. Woods B, Spector AE, Jones CA, Orrell M, Davies SP. Reminiscence therapy for dementia. Cochrane Database of Systematic Reviews. 2005;2:CD001120. 
19. Huang HC, Chen YT, Chen PY, et al. Reminiscence therapy improves cognitive functions and reduces depressive symptoms in elderly people with dementia: a metaanalysis of randomized controlled trials. J Am Med Dir Assoc. 2015;16:1087-94.

20. Low LF, Baker JR, Harrison F, et al. The Lifestyle Engagement Activity Program (LEAP): implementing social and recreational activity into case-managed home care. $\mathrm{J}$ Am Med Dir Assoc. 2015;16:1069-76.

21. Van Bogaert P, Van Grinsven R, Tolson D, et al. A feasibility trial of individual reminiscence based on the SolCos Model for people with mild to moderate dementia. JAMDA. 2013;14:528.e9-528.e13.

22. Van Bogaert P, Tolson D, Eerlingen R, et al. SolCos model-based individual reminiscence for older adults with mild to moderate dementia in nursing homes: a randomized controlled intervention study. Journal of Psychiatric and Mental Health Nursing. 2016;23:568-575.

23. Tolson D and Schofield I (2010) Scottish Football Museum Reminiscence Pilot. Project for People with Dementia: A Realistic Evaluation. Report for the Scottish Football Museum, Hampden Park, Glasgow. Available from: https://www.researchgate.net/publication/228970747_Scottish_Football_Museum_Re miniscence_Pilot_Project_for_People_with_Dementia_A_Realistic_Evaluation [accessed Jan 9, 2017].

24. Tolson D, Lowndes A, O’Donnell H, McGillivray D, Kinnaird L, Morley J (2013) Memories FC. Harnessing the Heritage of Football; Creating Meaningful Activities and Therapeutic Reminiscence Work with People with Dementia. Available from: http://www.ahrc.ac.uk/research/readwatchlisten/filmsandpodcasts/memoriesfcheritage offootball/ [accessed Jan 9, 2017].

25. Wingbermuehle C, Bryer D, Berg-Weger M, et al. Baseball reminiscence league: a model for supporting persons with dementia. J Am Med Dir Assoc. 2014;15:85-9.

26. Charleston, S. The English football ground as a representation of home. Journal of Environmental Psychology. 2009;29:144-150.

27. Giulianotti, R. Supporters, followers, fans, and flaneurs: a taxonomy of spectator identities in football. Journal of Sport and Social Issues. 2002;26:25-46.

28. Bale, J. The changing face of football: stadiums and communities. Soccer \& Society. 2000;1:91-101.

29. Tolson D, Schofield I. Football Reminiscence for men with dementia: lessons from a Realistic Evaluation. Nurse Inquiry. 2012;19:63-70

30. Reisberg B, Ferris SH, De Leon MJ, Crook T. The Global Deterioration Scale for assessment of primary degenerative dementia. Am J Psychiatry. 1982;139:1136-39.

31. Patton, MQ. Qualitative evaluation and research methods. 2nd ed. Thousand Oaks, CA, US: Sage Publications, Inc., 1990.

32. Nolan MR, Brown J, Davies S, et al. The Senses Framework: improving care for older people through a relationship-centred approach. Getting Research into Practice (GRIP), Series No. 2. University of Sheffield, 2006.

33. Nolan MR, Davies S, Brown J, et al. Beyond 'person-centred' care: a new vision for gerontological nursing. International Journal of Older People Nursing. 2004;13;45-53.

34. Watchman K, Tolson D, Gallagher N, et al. (2015) Football reminiscence for men with dementia in a care home. Hamilton: University of the West of Scotland. 
35. Gaventa J, Cornwall A. Power and Knowledge. In: Reason P, Bradbury H, eds. The SAGE handbook of Action Research Participative Inquiry and Practice. 2nd ed. Los Angeles, London, New Delhi, Singapore: SAGE Publications, 2008. 


\section{Appendix 1 Principles and Practice Guide for Developing Football-Focused Reminiscence with People with Dementia.}

Reproduced and adapted content is from the Scientific Report (Tolson et al. 2011) with permission from the Arts and Humanities Research Council UK.

\begin{tabular}{|c|}
\hline $\begin{array}{l}\text { Principles and Practice Guide to Delivery of } \\
\text { Football-Focused Reminiscence for People with Dementia }\end{array}$ \\
\hline $\begin{array}{l}\text { The aim of football reminiscence is to provide meaningful activity and social stimulation for people with } \\
\text { dementia. Reminiscence can be delivered on an individual or group basis. A benefit of group-based football } \\
\text { reminiscence is that it offers companionship, mutual support and a sense of belonging to football } \\
\text { enthusiasts. The following guidance is aimed at groups or organizations considering establishing a football } \\
\text { reminiscence program for people with dementia. As a starting point it should be recognized that } \\
\text { enthusiasm must be coupled with the necessary structures and resources to implement the appropriate } \\
\text { supporting policies and procedures referred to in this guide. }\end{array}$ \\
\hline
\end{tabular}

\section{Model of Delivery: Facilitator}

Reminiscence facilitation may be undertaken by health and social care practitioners and/or volunteers with appropriate dementia care skills, training and supervision. The therapeutic intention of the session will determine the required mix of practitioners and volunteers, and balance between dementia expertise and understanding of football.

The Alzheimer Scotland community delivery approach to football reminiscence, which has been informed through experience, research and knowledge exchange, is based on a volunteer-delivery model. Volunteers are matched on a one-to-one basis with a person with dementia, who is living in their own home or a care home. Alternatively volunteers can work with a Reminiscence Facilitator to deliver group-based sessions within a care home or a dementia-friendly community venue.

The volunteer who is working with an individual will do so in an imaginative way to create something which is a unique expression of each individual's football-related memories. Volunteers may also accompany the person to visit places that are meaningful to them, such as football grounds and football museums.

\section{The Reminiscence Venue (Community Group-Based Interventions)}

The community venue chosen for the group must possess the following attributes:

- The aesthetics and physical design of the facility should be dementia friendly.

- Football-related artefacts should be displayed to provide visual cues.

- Football displays should be easily set up before use and taken down after use.

- The facility should be large enough to accommodate between 6 and 12 people including wheelchair users, with appropriate furniture to engage in table-top activities.

- The same venue should be available at the same time each week for the duration of the program (for example at least 12 weeks).

- Transportation plans should be agreed and rehearsed to ensure that all participants arrive in time to attend to personal needs (e.g. toilet, rest) prior to the start of the reminiscence sessions.

\section{Facilitator Induction and Training}

There should be an initial introductory session to explain to new facilitators and helpers the ethos, structure and purpose of the program and provide a basic understanding of the nature of dementia. Training should aim to develop:

- An understanding of the purpose and benefit of reminiscence activity 
- An understanding of dementia

- Appreciation of person-centered approaches

- Understanding of the intervention approach and protocol

- Selection and effective use of archive materials

- $\quad$ Planning enjoyable activities appropriate to the person's abilities and interests.

\section{Structured Reminiscence Intervention (Group-Based)}

The following principles are based on findings from previous work and are recommended as they appear to enable people with dementia to engage in football reminiscence:

1. Group membership should be consistent and comprise between 4 and 12 people with dementia.

2. The same facilitator and helpers should manage the sessions.

3. An evaluation plan and methods should be agreed and appropriate consents obtained.

4. Twelve-week programs with weekly sessions allow for evaluation of individual benefit.

5. Sessions should be structured with a predictable format of activities to open and close the session. The structure should be agreed in advanced, informed through evidence and experience-based knowledge.

6. Activities should be varied at a pace appropriate to the group and individual responses and might include songs, photograph and visual image elicited reminiscence, artefact tactile or sensory stimulation of memories such as through smells and sounds. Creative arts and personal memorabilia can also be used.

\section{Eligibility Criteria}

Careful consideration needs to be given to the different challenges that individuals will face as their condition progresses. Published studies have focused on reminiscence interventions with people with mild to moderate dementia. A few papers suggest benefits for people with advanced dementia but no studies have included individuals at the terminal end of life stage. For community-based interventions thought needs to be given to the complexity of delivering interventions safely to a mixed group at different stages of dementia (see below). It is advisable to develop clear admission criteria and to factor this into decision making about staff-to-participant ratios and to the planning of appropriate activities.

\section{Keeping people with dementia safe}

All potential volunteers must be subject to a disclosure check before they are matched with a person with dementia in the case of individual sessions, or before they are in charge of a group. A code of practice should be followed that includes confidentiality about the individual's and family members' circumstances and respect for the security of property and belongings. For volunteers who work within care-home environments the privacy and safety of other residents must also be highlighted. People with dementia are vulnerable adults and there should be a mechanism through which the volunteer might seek guidance should they have a particular concern for a person's welfare.

\section{Monitoring and evaluation}

The benefit of football reminiscence will vary and may be difficult to quantify. Individual experience is central; feedback from the person with dementia and their caregiver is a key source of identifying the impact of participating in football reminiscence. The behavior and mood of the person with dementia are also important factors in understanding the impact; facial expression, engagement and body language are key factors. 
Table 1. Results for participants of the Spanish and the Scottish projects.

\section{Reported impacts on participants}

\section{Spanish Project}

* Participants, staff, family members and volunteers reported a positive impact on mood, and they expressed positive feelings of joy and psychological wellbeing.

* Staff and family members reported an increase in selfesteem of participants; they felt valued and useful sharing their knowledge and experience.

* Staff and family members observed and reported that participants increased their communicative (more talkative) and cognitive abilities (memory and attention) during the sessions.

* Decrease in social isolation through improvements in socialization was reported and observed by all involved agents, consisting of an increase in quantity and quality of social interactions between participants during and between sessions. Participants living in the same nursing home got to know each other through the program.

* Positive displays of anticipation were observed by staff and family members consisting of participants waiting for weekly sessions with enthusiasm.

* Participants increasingly showed engagement in the group dynamics developing a strong sense of belonging.

\section{Scottish Project}

* Participants exhibited increased self-awareness, evidenced by two of the men bathing before attending whereas typically they refused to do so, and all dressing willingly and smartly in preparation for the sessions, suggesting the potential for improved dementia symptomology.

* Participants displayed pride at being positioned as experts; some of the men had extensive football knowledge even beyond that of the facilitator.

* Improved sleep was reported for two of the men after football reminiscence sessions, with one acknowledging that the travel involved and being outdoors for a period of time may have also been a contributory factor.

* Staff and family members reported that participants increased communication on the "football days" compared to other days of the week. This was unexpected as all of the men were considered to have declining abilities to converse or use verbal expression.

* Staff reported that participants were sometimes awake earlier on the intervention days and showed other positive displays of anticipation including visible signs of enjoyment at arrival and warmth of greeting the other men and the facilitator. 


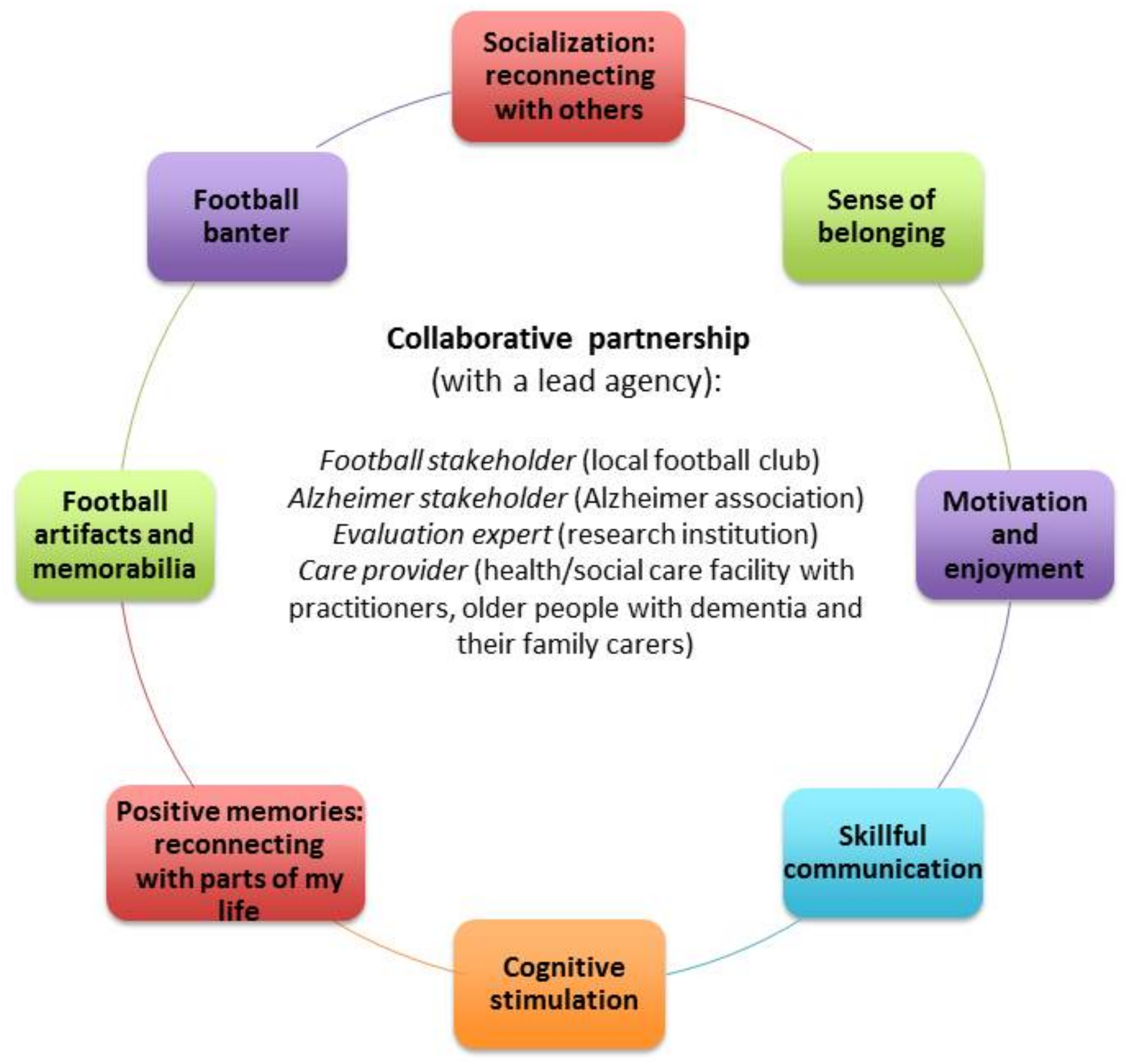

